\title{
Model-Based Specification for System Development with Suppliers
}

\author{
Phanikrishna Thota ${ }^{1(凶)}$, Simon Hancock ${ }^{1}$, Mario Noriega-Fogliani ${ }^{2}$, \\ and Rodrigo Jimenez ${ }^{2}$ \\ 1 Airbus Operations, Ltd., Pegasus House, Filton, Bristol BS34 7PA, UK \\ \{Phanikrishna. Thota, Simon. Hancock\}@airbus.com \\ 2 AKKA, New Filton House, 20 Golf Course Lane, Bristol BS34 7QW, UK
}

\begin{abstract}
Authoring, validating and communicating system requirements becomes even more important when the development is contracted to an external supplier. This paper illustrates the process of creating and communicating a model-based specification as an alternative to a text-based one in a real-world project called OnePRESS, a digital platform to monitor aircraft tyre inflation pressure.

The logical part of the specification is broken down functionally and allocated to the top-level product breakdown. The interfaces for each such function are drawn while considering the actual design as a black-box. An executable Simulink model is then created using library blocks with unique identification, where each library block forms a requirement and are configuration-controlled. A test model is assembled by combining the library blocks and Operational Scenarios are used to validate the assembled test model. The validated model and the associated libraries are delivered to the supplier, and form the basis for the system development.
\end{abstract}

